\title{
Brief 11: Grete Hermann an ihre Mutter [Auguste Hermann (geb. Leipoldt)] über ihre Gespräche mit Heisenberg in Leipzig und andere philosophische Arbeitsberichte $(24.6 .1934)^{1}$
}

\section{VI. 34}

Ich habe hier eine philosophische Diskussion vor mit Dubislav und seinen Schülern. Das sind so Philosophen, die wo[hl] nicht an die Vernunft glauben, sondern nur ihren Sinnen trauen wollen. Da werde ich versuchen, ihnen von meinen Leipziger Ergebnissen zu erzählen, und darüber hinaus, was es mit der Vernunft und ihrer Erkenntnis vom Kausalgesetz auf sich hat. Das wird dann einen Kampf geben, wie Du Dir denken kannst. ... Im Juli gehe ich vielleicht ans Institut von Niels Bohr. ... ${ }^{3}$ event. fahre ich auch doch noch auf ein paar Wochen nach Leipzig zurück und mache das Seminar noch bis zu Ende mit.

1 Abschrift vermutlich von Carl Heinrich Hermann

2 In der Abschrift als Auslassung gekennzeichnet

3 In der Abschrift als Auslassung gekennzeichnet

(C) Springer Fachmedien Wiesbaden GmbH, ein Teil von Springer Nature 2019

K. Herrmann (Hrsg.), Grete Henry-Hermann: Philosophie - Mathematik-

Quantenmechanik, Frauen in Philosophie und Wissenschaft. Women

Philosophers and Scientists, https://doi.org/10.1007/978-3-658-16241-2_33 